Abstract PS8:171 Table 1 Disfuncion endotelial

\begin{tabular}{|c|c|c|c|}
\hline & No damage & Damage & \\
\hline Statin & $10,127 \%(8)$ & $22,5 \%(9)$ & $\mathrm{p}=0,122$ \\
\hline Antiplatelet agents & $21,52 \%(17)$ & $37,5 \%(15)$ & $p=0,101$ \\
\hline Smoker-Former smoker & $22,78 \%(8)$ & $25,0 \%(10)$ & $p=0,943$ \\
\hline Obesity & $20,78 \%(16)$ & $10,0 \%(4)$ & $\mathrm{p}=0,226$ \\
\hline Dyslipemia & $13,92 \%(11)$ & $32,5 \%(13)$ & $p=0,032$ \\
\hline Hypertension & $13,92 \%(11)$ & $47,5 \%(19)$ & $p<0,001$ \\
\hline Age at diagnosis & $30,94 \pm 9,68$ & $37,92 \pm 15,79$ & $\mathrm{p}=0,003$ \\
\hline Evolution time & $12,96 \pm 9,88$ & $18,73 \pm 13,13$ & $p=0,008$ \\
\hline SLEDAI & $2,47 \pm 2,79$ & $2,38 \pm 2,57$ & $p=0,856$ \\
\hline Corticoids & $39,24 \%(31)$ & $35,0 \%(14)$ & $\mathrm{p}=0,802$ \\
\hline Antimalarial drugs & $89,873 \%(71)$ & $87,5 \%(35)$ & $\mathrm{p}=0,759$ \\
\hline Inmunosuppressive & $35,443 \%(28)$ & $30 \%(12)$ & $\mathrm{p}=0,698$ \\
\hline Corticoids treatment previously & $53,165 \%(42)$ & $45 \%(18)$ & $p=0,517$ \\
\hline Cholesterol & $182,0933,49$ & $195,7035,45$ & $\mathrm{p}=0,042$ \\
\hline HDL cholesterol & $65,10 \pm 18,18$ & $56,85 \pm 15,78$ & $p=0,016$ \\
\hline LDL cholesterol & $100,61 \pm 28,93$ & $118,17 \pm 30,13$ & $p=0,003$ \\
\hline Triglycerides & $82,18 \pm 43,28$ & $106,38 \pm 49,76$ & $p=0,007$ \\
\hline
\end{tabular}

\section{Poster session 9: Miscelleanea}

\section{PS9:172 CHARACTERISTICS OF LUPUS NEPHRITIS IN SAUDI LUPUS PATIENTS: A RETROSPECTIVE OBSERVATIONAL STUDY}

I Al-Homood, A Almasmaly, M Albirdisi. King Fahad Medical City, Medical Specialities Department, Rheumatology Section, Riyadh, Saudi Arabia

\subsection{6/lupus-2018-abstract.215}

Objective The aim of this study is to evaluate the prevalence, clinical, laboratory and histological types of lupus nephritis (LN) among Saudi patients.

Methods This is a retrospective study that done at King Fahad Medical City, Riyadh, Saudi Arabia, where clinical and laboratory data collected for a period from 2011-2016. All patients fulfilled the Systemic Lupus International Collaborating Clinics classification criteria (SLICC).

Results Out of 112 patients, 103 (92.0\%) females, 9 (8.0\%) males, mean age $34(33.87 \pm 10.42)$ years. The mean of initial proteinuria level was $0.72 \mathrm{gm}(0.72 \pm 1.34)$ and the mean serum creatinine $34.00(82.33 \pm 83.88)$. The most common clinical characteristics were skin rash $70 \%$ and photosensitivity $61 \%$.

With regards to comorbidities; Hypertension was found in 31 (27.7\%), Hyperlipidemia in $16(14.3 \%)$ and Diabetes mellitus in $12(10.7 \%)$ of SLE patients.
Laboratory investigations revealed $67.0 \%$ had a positive ANA, $60.7 \%$ positive Anti DsDNA, $27.7 \%$ positive aPL, 17.9\% positive Anticardiolipin igG, $18.8 \%$ positive Anticardiolipin IgM, and B2glycoprotein IgG was positive in $8.9 \%$ of patients.

$44(39.8 \%)$ out of 112 patients had a kidney biopsy and the histological types were as follow; Focal segmental glomerulosclerosis (FSGS) 2.2\%, class I 2.2\%, class II 6.8\%, class III 27.2\%, class IV 43.1\%, V 6.8\%, minimal change disease (MCD) 2.2\%, Thrombotic Microangiopathy (TMA) 2.2\%, non-specific 2.2\% Normal 2.2\%, poor biopsy $2.2 \%$.

Conclusion

- The most notable clinical manifestations were skin rash $70 \%$ followed by photosensitivity 61\% and arthralgia 39\%.

- Patients with Hypertension, hyperlipidemia and diabetes mellitus had a higher risk of developing $\mathrm{LN}$ at any stage by 10\%-28\%.

- The commonest class of lupus nephritis among our patients as proven by kidney biopsy is class IV $43.1 \%$ followed by class III 27.2\%.

\section{PS9:173 TWO DECADES OF NEUROLUPUS}

ASX Pires, M Cunha, M Formigo, S Freitas, G Alves, J Cotter. Hospital da Senhora da Oliveira, Guimarães, Portugal

10.1136/lupus-2018-abstract.216 
Systemic lupus erythematosus (SLE) is a clinically heterogeneous multi-system disease, that is characterised by the presence of autoantibodies directed against nuclear antigens. The most common manifestations include rash, arthritis, fatigue, but also anaemia, thrombocytopenia, nephritis and neurologic symptoms. The latter remain one of the most challenging of all classification criteria, since lupus patients might present with a multiple array of psychiatric and neurologic symptoms, such as depression, anxiety and personality disorder, dementia, asseptic meningitis, demyelinating syndromes, and cerebrovascular disease.

The aim of this project was to understand the prevalence, morbidity and outcome associated with non-primary neuropsychiatric Lupus erythematosus systemic at our auto-immunity diseases centre. Clinical records of 128 patients followed at the clinic between January 1993 and December 2016 were read, and national registry of Auto-immune diseases was consulted in order to characterise the Lupus cohort.

From all 137 patients assessed, average age was 49, with 93\% of all patients being females. The most frequent clinical criteria were malar rash in $72 \%$, arthritis in 51\%, hematologic disturbances in 43\%, from which the most frequent was lymphopenia.

Amongst all patients, only $6,6 \%$ of patients $(n=9)$ presented with severe non-thrombotic neurologic impairment, with one patient presenting with trigeminal neuralgia, 5,8\% of patients presenting with magnetic ressonance imaging compatible with cerebritis, with three of the patients overlapping with seizures. However, minor neuropsychiatric impairment was very common, with headache being the most predominant complaint $(68,4 \%)$, as well as anxiety $(77 \%)$. All of patients were on a low dose corticosteroid regimen.

Diffuse neuropsychiatric manifestations of SLE remain a diagnostic challenge, because it is very difficult for the physician to understant whether these are caused by SLE or psychological reactions to the stress of coping with a major chronic systemic illness.

\section{PS9:174 PREVALENCE OF VASCULAR RISK FACTORS IN A COHORT STUDY OF PATIENTS IN FOLLOW-UP IN A UNIT OF AUTOIMMUNE DISEASES IN A 3TH LEVEL HOSPITAL IN SPAIN}

'A Martínez Zapico, ²Al Pérez Álvarez, 'L Caminal Montero, 'B Díaz López, ${ }^{2} \mathrm{~L}$ Benavente Fernández, ${ }^{1} \mathrm{R}$ Gómez de la Torre, ${ }^{1} \mathrm{D}$ Colunga Argüelles, ${ }^{3} \mathrm{~J}$ Rodríguez Carrio, ${ }^{3} \mathrm{P}$ López Suárez, ${ }^{3} \mathrm{~A}$ Suárez Díaz. ${ }^{1}$ Servicio de Medicina Interna. Hospital Universitario Central de Asturias, Oviedo- Asturias, Spain; ${ }^{2}$ Servicio de Neurología. Hospital Universitario Central De Asturias, Oviedo-Asturias, Spain; ${ }^{3}$ Área de Inmunología. Departamento de Biología Funcional. Universidad de Oviedo, Oviedo-Asturias, Spain

\subsection{6/lupus-2018-abstract.217}

Objectives Lupus systemic erythematosus is characterised by an increasing risk of premature cardiovascular disease (CVD). CVD is one of the most common causes of death in SLE. Subclinical atherosclerosis in comparison to general population is also more prevalent, especially the presence of plaques at the carotid level, as well as thickening of the carotid intima.

The aetiology of atherosclerotic disease is completely unknown. It involves: traditional risk factors (age, male gender, smoking, diabetes, hypertension, dyslipidemia, obesity) as well as risk factors related to the disease itself and the treatments used.

Methods A cross-sectional study was carried out from March to November 2015. 119 patients $(94,1 \%$ women) were

\begin{tabular}{l} 
Abstract PS9:174 Table 1 \\
\begin{tabular}{|ll|}
\hline Vascular risk factors. & Vascular risk factors \\
\hline Statin & $14,29 \%$ \\
\hline Hypotensive treatment & $29,41 \%$ \\
\hline Antiplatelet agents & $26,89 \%$ \\
\hline Smoker- Former smoker & $23,53 \%-19,33 \%$ \\
\hline Obesity & $17,09 \%$ \\
\hline Dislipemia & $20,17 \%$ \\
\hline Hypertensión & $25,21 \%$ \\
\hline Diabetes & 0 \\
\hline Age at diagnosis & $33,29 \pm 12,46$ years \\
\hline Evolution time & $14,89 \pm 11,36$ years \\
\hline SLEDAl & $2,44 \pm 2,71$ \\
\hline Corticoids & $37,93 \%$ \\
\hline Antimalarial drugs & $89,08 \%$ \\
\hline Inmunosuppressive & $32,76 \%$ \\
\hline Corticoids treatment previously & $50,42 \%$ \\
\hline Cholesterol & $186,66 \pm 34,62 \mathrm{mg} / \mathrm{dl}$ \\
\hline HDL cholesterol & $62,33 \pm 17,78 \mathrm{mg} / \mathrm{dl}$ \\
\hline LDL cholesterol & $106,51 \pm 30,38 \mathrm{mg} / \mathrm{dl}$ \\
\hline Triglycerides & $90,31 \pm 46,78 \mathrm{mg} / \mathrm{dl}$ \\
\hline
\end{tabular} \\
\hline
\end{tabular}

recruited from consultation at the Systemic Autoimmune Diseases Unit for a routine medical check. Clinical data on the disease (from diagnosis to the time of inclusion in the study) were obtained by reviewing the medical history. Data were collected about:

- Traditional vascular risk factors.

- Risk factors related to the disease: Age at diagnosis, time of disease progression, SLEDAI.

- Treatment performed.

- Lipid profile.

Results View table 1. Hypertension is one of the classic risk factors attributed to the disease. In our study the prevalence is $25 \%$, similar to the one found in other studies in literature. Likewise, the percentage of patients receiving antihypertensive therapy is higher than that of hypertensive patients. This can be explained by the use of antihypertensives for antiproteinuric purposes in patients with nephropathy. Unless contraindicated, most patients received antimalarials and the use of corticosteroids is still important.

Conclusions Once the vascular risk factors have been identified, strict control of these factors is important. The realisation of cardiosaludable diet and regular aerobic exercise, since this constitutes the most effective form for its control. In addition, a rapid and long-term remission of the inflammatory activity of the disease should be achieved, avoiding high doses of oral glucocorticoids, thus avoiding its side effects. The decrease in the activity of the disease allows to do physical exercise, which would have beneficial consequences controlling the body mass index and hypertension.

\section{PS9:175 TWO DECADES OF LUPUS NEPHRITIS}

M Cunha, SX PIRES, M Formigo, S Freitas, G Alves, C Jorge. Hospital da Senhora da Oliveira, Guimarães, Portugal

10.1136/lupus-2018-abstract.218 